\title{
Atenuação de Reflexões Múltiplas na Região do Talude Continental utilizando De- convolução Iterativa
}

Felipe Vidal Fernandes; Milton José Porsani, CPGG/UFBA e INCT-GP/CNPq/MCT e Paulo Espinheira Menezes de Melo, CPGG/UFBA e INCT-GP/CNPq/MCT

Copyright 2010, SBGf - Sociedade Brasileira de Geofísica Este texto foi preparado para a apresentação no IV Simpósio Brasileiro de Geofísica, Brasília, 14 a 17 de novembro de 2010. Seu conteúdo foi revisado pelo Comitê Técnico do IV SimBGf, mas não necessariamente representa a opinião da SBGf ou de seus associados. É proibida a reprodução total ou parcial deste material para propósitos comerciais sem prévia autorização da SBGf.

\section{ABSTRACT}

The seismic reflection is largely used by oil companies in imaging of the surface offshore basin. However, the trace seismic has intrinsic noises in seismic acquisition. From these, we can mention those inherent trapping seismic energy due to the strong impedance contrast between two means, this energy reverberating, generating multiple events. In this work, we use the method of Multichannel Predictive Deconvolution using Lp norm. This type of filtering applied on common offset sections combined with the method of MMO correction, to correct the periodicities fault in the offsets farthest, in attenuation of multiple reflection of seabed in the region of continental slope. For such, seismic data of marine seismic line was conducted, by the use of the seismic processing software Focus and SU-CWP, and FORTRAN routines, developed in CPGG-UFBA, for multiple filtering of 214-RL-0266 of Jequitinhonha basin.

\section{INTRODUÇÃO}

Os métodos para a atenuação das reflexões múltiplas baseiam-se principalmente na periodicidade ou na diferença de moveout entre múltiplas e primárias (Yilmaz, 1987). Dentre os que se baseiam na diferença de moveout podemos citar a filtragem $\mathrm{f}-\mathrm{k}$, Radon e até mesmo o empilhamento CMP. Há ainda aqueles que são baseados na equação da onda, que modelam ou invertem o campo de onda registrado, simulando as múltiplas e efetuando a sua remoção (Santos Jr., 2002).

A deconvolução do pulso sísmico e de eventos múltiplos são etapas importantes dentro do processamento sísmico. A deconvolução do pulso sísmico objetiva aumentar a resolução temporal dos sismogramas, permitindo uma melhor identificação das superfícies refletoras em subsuperfície. Já a deconvolução de reflexões múltiplas, a chamada deconvolução preditiva, visa predizer e atenuar eventos periódicos contidos num sismograma, tais como múltiplas do fundo do mar.
O método da deconvolução iterativa é baseado na norma $L_{p}\left(D I L_{p}\right)$, diferentemente dos métodos convencionais que utilizam a norma $L_{2}$. Os erros de predição do traço sísmico, devido ao filtro de predição maior que a unidade, são elevados a um expoente relacionado à norma em uso, definindo assim um relacionamento não linear entre os coeficientes do filtro preditivo e o resultado da deconvolução. Através da expansão de Taylor com aproximação de primeira ordem, obtém-se um sistema de equações lineares e, dessa forma, obtém-se uma solução. Este método não requer que o pulso satisfaça a premissa de fase mínima. O presente trabalho apresenta alguns resultados da aplicação desta metodologia na filtragem de múltiplas associadas à região da quebra da plataforma continental de uma linha sísmica da bacia do Jequitinhonha, os quais são comparados e discutidos frente àqueles obtidos com o método convencional.

\section{MODELO CONVOLUCIONAL COM MÚLTIPLAS}

O método da Sísmica de Reflexão baseia-se na reflexão de pulsos sísmicos refletidos em interfaces entre meios que possuem contraste de impedância acústica. Quando esse contraste é muito grande, a energia sísmica pode ficar aprisionada, reverberando entre os dois meios, gerando então o ruído na seção sísmica denominado de reflexão múltipla.

Um exemplo prático e que será abordado neste trabaIho são as múltiplas associadas ao assoalho oceânico. Isto porque o contraste de impedância entre as interfaces água-ar e o fundo oceânico são suficientemente fortes para que a energia sísmica gerada durante as aquisições sísmicas marítimas, reverbere sucessivamente na lâmina d'água presente acima do assoalho, causando forte presença das múltiplas nos dados sísmicos marinhos (Figura 1 .

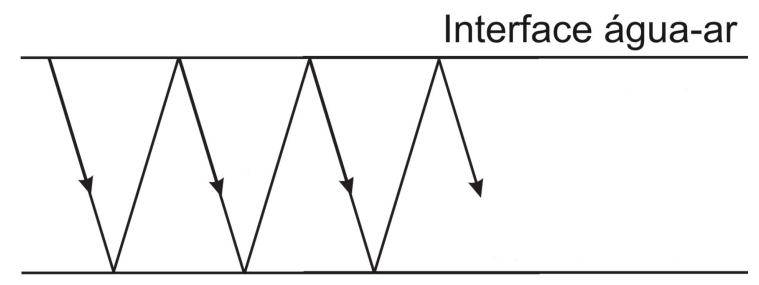

Fundo oceânico

Figura 1: Reverberação da energia sísmica na lâmina d'água. 
Com base no modelo convolucional do traço sísmico com múltiplas pode ser assim descrito:

$$
x(t)=p(t) * e(t) * m(t) .
$$

Onde $x(t)$ é o traço sísmico, $p(t)$ é o pulso sísmico considerado invariante no tempo e $e(t)$ representa a resposta impulsiva da Terra, que inclui as primárias e as múltiplas internas (Yilmaz, 1987), e $m(t)$ é a seqüência geradora de múltiplas do fundo do mar que desejamos remover.

\section{DECONVOLUÇÃO PREDITIVA COM NORMA $L_{P}$}

Seguindo o procedimento proposto por Porsani et al. (2001), Melo (2002) e Fernandes (2010), podemos definir a função objetivo em termos da norma $L_{p}$ como segue:

$$
Q(\mathbf{a}, p)=\sum_{t=0}^{M+N}\left\{\left[\left(e_{t}\right)^{2}\right]^{1 / 2}\right\}^{p}
$$

onde $e_{t}$ representa o erro com distância de predição $L$ dado por:

$$
e_{t+L}=\left(\begin{array}{llllll}
x_{t+L} & x_{t+L-1} & \cdots & x_{t} & \cdots & x_{t-N+1}
\end{array}\right)\left(\begin{array}{c}
1 \\
0 \\
\vdots \\
-a_{1} \\
\vdots \\
-a_{N}
\end{array}\right) .
$$

O vetor erro é dado por:

$$
\mathbf{e}=\mathbf{x}-\mathbf{X} \mathbf{a}_{k} .
$$

sendo a matriz $\mathbf{X}$, Toeplitz, formada pelos elementos $\mathbf{x}_{t}$ do vetor $\mathrm{x}$ e $\mathrm{a}_{0}$ o filtro de Wiener-Levinson usado para iniciar o método.

Deixemos:

$$
f(\mathbf{a}, p, t)=\left[\left(e_{t}^{2}\right)^{1 / 2}\right]^{p / 2}
$$

e expandindo a equação acima por série de Taylor em torno do modelo corrente $\mathbf{a}_{k}$, e tomando a aproximação de primeira ordem, obtém-se:

$$
\begin{gathered}
f(\mathbf{a}, p, t) \cong \tilde{f}(\mathbf{a}, p, t)=f\left(\mathbf{a}_{\mathbf{k}}, p, t\right)+ \\
\left.\frac{p}{2}\left[\left(e_{t}^{2}\right)^{1 / 2}\right]^{p / 2-1}\left(e_{t}\right) \frac{\partial e_{t}}{\partial \mathbf{a}}\right|_{\mathbf{a}_{k}}\left(\mathbf{a}-\mathbf{a}_{k}\right) .
\end{gathered}
$$

A aproximação por série de Taylor faz com que a função $f(\mathbf{a}, p, t)$ que antes era de natureza não linear, se torne agora uma função linear.

A equação anterior pode ser representada de forma mais compacta,

$$
\tilde{\mathbf{f}}_{p k}=\mathbf{f}_{p k}-\mathbf{F}_{p k} \mathbf{X} \Delta \mathbf{a}
$$

onde os elementos $\mathbf{F}_{i} i$ da matriz diagonal $\mathbf{F}_{p} k$ são dados por:

$$
\begin{gathered}
F_{i i}=\frac{p}{2}\left|\left(e_{i}^{2}\right)^{1 / 2}\right|^{p / 2-1} e_{i}, \\
f_{i}=\left|\left(e_{i}^{2}\right)^{1 / 2}\right|^{p / 2},
\end{gathered}
$$

e $\mathbf{e}_{t}^{2}$ representa a amostra na posição $t=i \Delta t$ do traço deconvolvido com o filtro preditivo de erro (1 $\mathbf{a}_{k}^{T}$ ).

O vetor $\Delta a$ pode ser calculado de forma a minimizar a forma quadrática,

$$
\tilde{Q}(\mathbf{a}, p)=\tilde{\mathbf{f}}_{p}^{T} \tilde{\mathbf{f}}_{p}
$$

Derivando com relação aos parâmetros $a_{j}, j=1, \ldots, \mathrm{N}$, obtém-se as equações normais (ENs):

$$
\left(\mathbf{X}^{T} \mathbf{F}_{p k}^{2} \mathbf{X}\right) \Delta \mathbf{a}=\mathbf{X}^{T} \mathbf{F}_{p k} \mathbf{f}_{p k} .
$$

Resolvendo a equação acima para o vetor $\Delta a$ podemos atualizar os coeficientes do filtro por:

$$
\mathbf{a}_{k+1}=\mathbf{a}_{k}+\Delta \mathbf{a} .
$$

De maneira análoga ao monocanal, podemos obter as ENs para o caso multicanal.

\section{METODOLOGIA/ PROBLEMA INVESTIGADO}

A deconvolução preditiva é um método estatístico que convencionalmente utiliza o processo de filtragem desenvolvido por Wiener-Levinson (normal $L_{2}$ ). Este se baseia na periodicidade das múltiplas, a qual é observada ao aplicarmos ao dado, no domínio CMP, uma correção de MMO (Multiple Moveout) e organizarmos no domínio do offset-comum, o que diminui também o problema da não estacionariedade (Maciel et al., 2003). Tal correção nada mais é que uma correção de NMO ( Normal Moveout) utilizando a velocidade da múltipla que se deseja suprimir. Com a aplicação do MMO em dados registrados sobre um fundo do mar horizontal tanto a reflexão primária quanto as múltiplas do fundo do mar tornamse horizontais e periódicas com distância de predição igual ao tempo zero-offset da primária (na Figura 2 podese verificar a correção de MMO). Porém, esses eventos não formam uma série estacionária no espaço e nem no tempo devido ao estiramento produzido pelo MMO. Por isso, a organização dos traços para o domínio do afastamento comum que tenta resolver o problema da não estacionaridade das múltiplas no espaço.

Para a execução dos processos que antecedem a correção de $\mathrm{MMO}$ (que se fazem necessários para um correto tratamento dos dados), como geometria, edição e filtragem, utilizamos o software de processamento sísmico Focus. Para a aplicação da deconvolução e a organização dos traços no domínio do afastamento comum foram utilizados o Seismic Unix e rotinas Fortran desenvolvidas no CPGG - UFBA. 


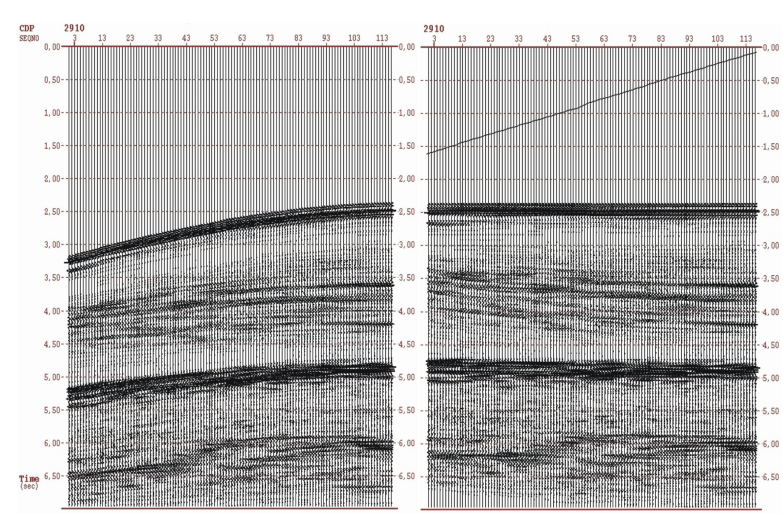

Figura 2: Supergather em uma família CMP antes e após a aplicação da correção de MMO.

\section{RESULTADOS}

Após a realização da correção de $\mathrm{MMO}$ e da organização dos traços no domínio do afastamento comum, sucessivos testes foram realizados com objetivo de determinar os parâmetros ótimos da Deconvolução Preditiva Multicanal (DPM): coeficientes do filtro (ncf), a distância de predição (L), a norma Lp e o número de iterações (n). Os melhores resultados foram obtidos para ncf $=0.16 \mathrm{P}$; $\mathrm{L}=0.92 \mathrm{P}(\mathrm{P}=$ período da múltipla $) ; \mathrm{L}=1.5$ e $\mathrm{n}=10$.

Posteriormente a aplicação da DPM faz-se necessário a aplicação da correção inversa de MMO, na qual utiliza as mesmas velocidades que foram selecionadas durante a aplicação da correção de MMO. No entanto, esta correção não utiliza as verdadeiras velocidades das camadas, de maneira que esta é necessária apenas para tornar as reflexões múltiplas periódicas, fazendo com que a aplicação da técnica da deconvolução seja mais efetiva. Deve-se aplicar a correção inversa de MMO para deslocar as amostras para as suas posições temporais verdadeiras, para poder realizar a análise de velocidade.

A Figura 3 e 4 demostra a aplicação da correção inversa de MMO em uma família CMP após a aplicação da DPM com norma $L_{2}$ e $L_{p}$.

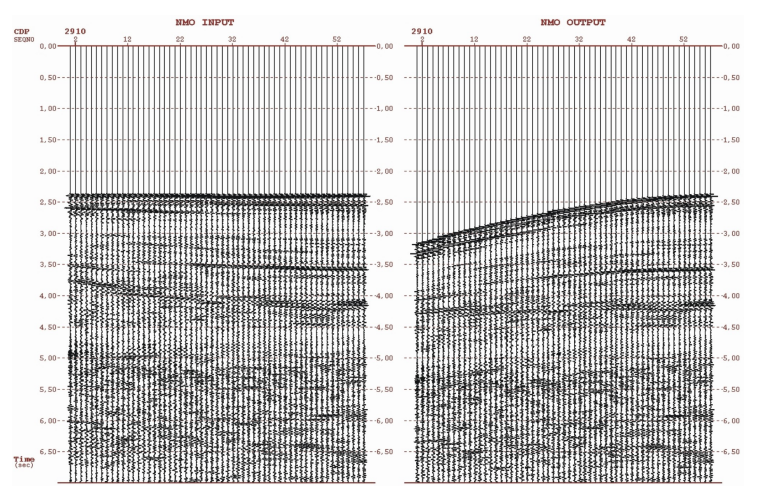

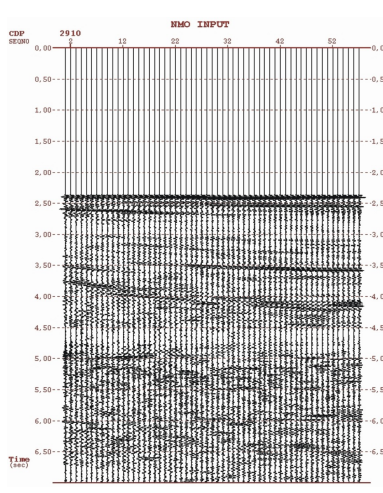

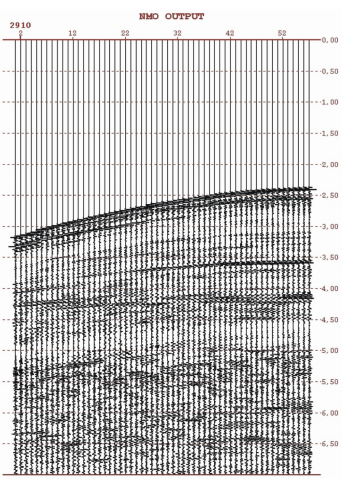

Figura 4: CMP 2910 antes e após a correção inversa de MMO da DPM- $L_{p}$.

Então, ambos os métodos atuaram de maneira satisfatória na atenuação dos ruídos coerentes denominados de múltiplas, porém o método proposto da DPM- $L_{p}$ atuou melhor, mesmo que em pequenos detalhes, do que o método convencional da DPM- $L_{2}$, como pode ser observado, por exemplo, nas seções de afastamento comum (Figuras 5, 6] 7 e 8.

\section{DISCUSSÃO E CONCLUSÕES}

A aplicação da deconvolução preditiva multicanal (DPM) em seções de afastamento comum forneceu resultados satisfatórios na atenuação das reflexões múltiplas do fundo do mar na região do Talude Continental.

A correção de MMO antes da aplicação da DPM é importante porque consegue fazer com que as reflexões múltiplas ocorram com certa periodicidade, fazendo com que o método de filtragem preditiva atue de forma mais efetiva. A aplicação do método sobre traços de mesmo offset, ou seja, painéis de afastamento comum, também é importante porque ajuda a reduzir o efeito da variação na forma do pulso sísmico devido à correção de NMO, variável ao longo dos offsets.

A deconvolução preditiva com norma $L_{p}$ mostrou-se mais eficaz do que a com norma $L_{2}$ na maior parte do dado, neste sentido a análise de velocidade para a correção de $\mathrm{MMO}$, bem como a determinação dos parâmetros da deconvolução (ncf, L, Lp, n) que melhor se ajustavam as amostras foram fundamentais para o sucesso da deconvolução.

A DPM mostrou-se eficiente na atenuação de eventos múltiplos na região do talude continental (Bacia do Jequitinhonha), onde o assoalho oceânico é inclinado. Entendemos estes resultados como preliminares e sugerimos que trabalhos futuros sejam realizados para corroborar os resultados aqui apresentados.

Figura 3: CMP 2910 antes e após a correção inversa de MMO da DPM- $L_{2}$. 
AGRADECIMENTOS

Agradecemos ao INCT-GP/CNPq/MCT e seus patrocinadores e colaboradores: PETROBRAS, CNPQ/PRONEX/ FAPESB, ANP, FINEP-Rede Cooperativa NNE em Geofísica de Exploração. Agradecemos também às empresas Paradigm Geophysical e à Landmark pela licenças educacionais dos softwares de processamento sísmico.

\section{REFERÊNCIAS}

Fernandes, F. V., 2010, Atenuação de reflexões múltiplas na região do Talude continental utilizando deconvolução iterativa: Trabalho de Graduação, Universidade Federal da Bahia, Salvador - BA.

Porsani, M. J., Niwas, S. and Ferreira, N. R., 2001, A robust inversion of vertical electrical sounding data using a multiple reweighte least-squares method: Geophysical Prospecting, 49, no. 2, 255-264.

Melo, P. E. M., 2002, Deconvolução iterativa do pulso sísmico utilizando norma $L_{p}$ : Master's thesis, Universidade Federal da Bahia, Salvador - BA.

Maciel, R. C., Melo, P. E. M. and Porsani, M. J., 2003, Deconvolução de reflexões múltiplas do fundo do mar utilizando um método iterativo multicanal: Resumos expandidos, Congresso Internacional Brasileiro de Geofísica, Rio de Janeiro - RJ.

Santos Jr., A., 2002, Deconvolução multicanal utilizando um algoritmo tipo Wiener-Levinson: Master's thesis, Universidade Federal da Bahia, Salvador - BA.

Yilmaz, Ö., 1987, Seismic Data Processing: Society of Exploration Geophysicists, Tulsa - Oklahoma. 
Atenuação de Reflexões Múltiplas utilizando DILp

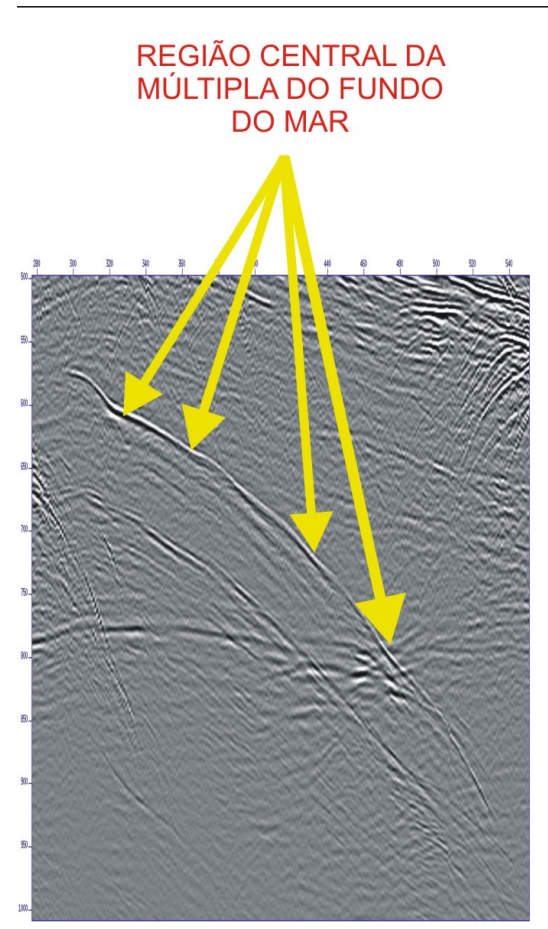

(a)

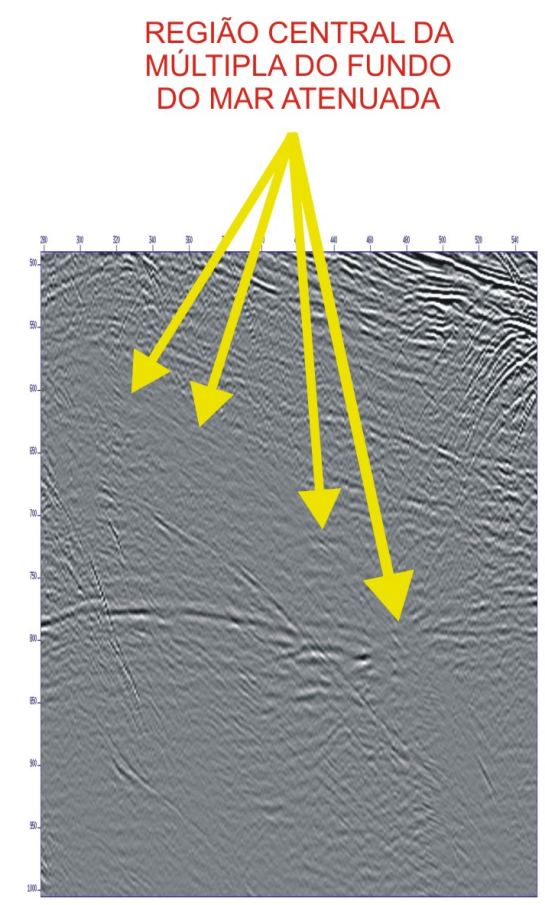

(b)
REGIÃO CENTRAL DA

MÚLTIPLA DO FUNDO

DO MAR ATENUADA

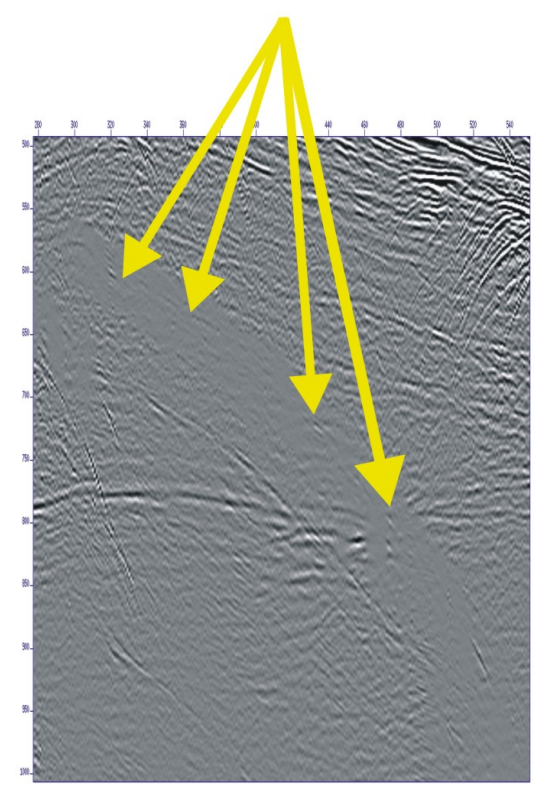

(c)

Figura 5: Painel de afastamento comum com zoom (a) antes e após a aplicação da (b) DPM- $L_{2}$ e (c) DPM- $L_{p}$.

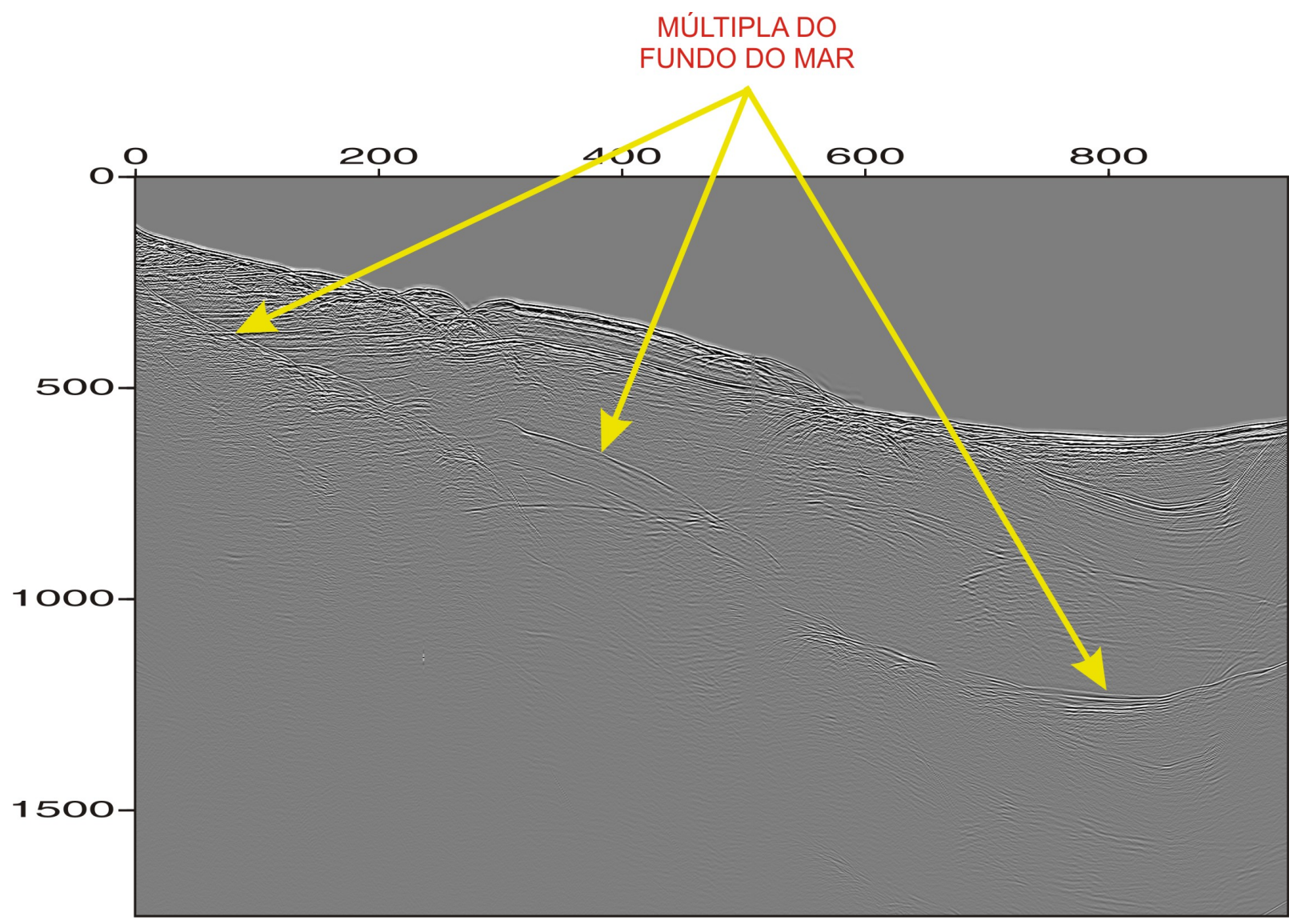

Figura 6: Painel de afastamento comum da Bacia do Jequitinhonha. 


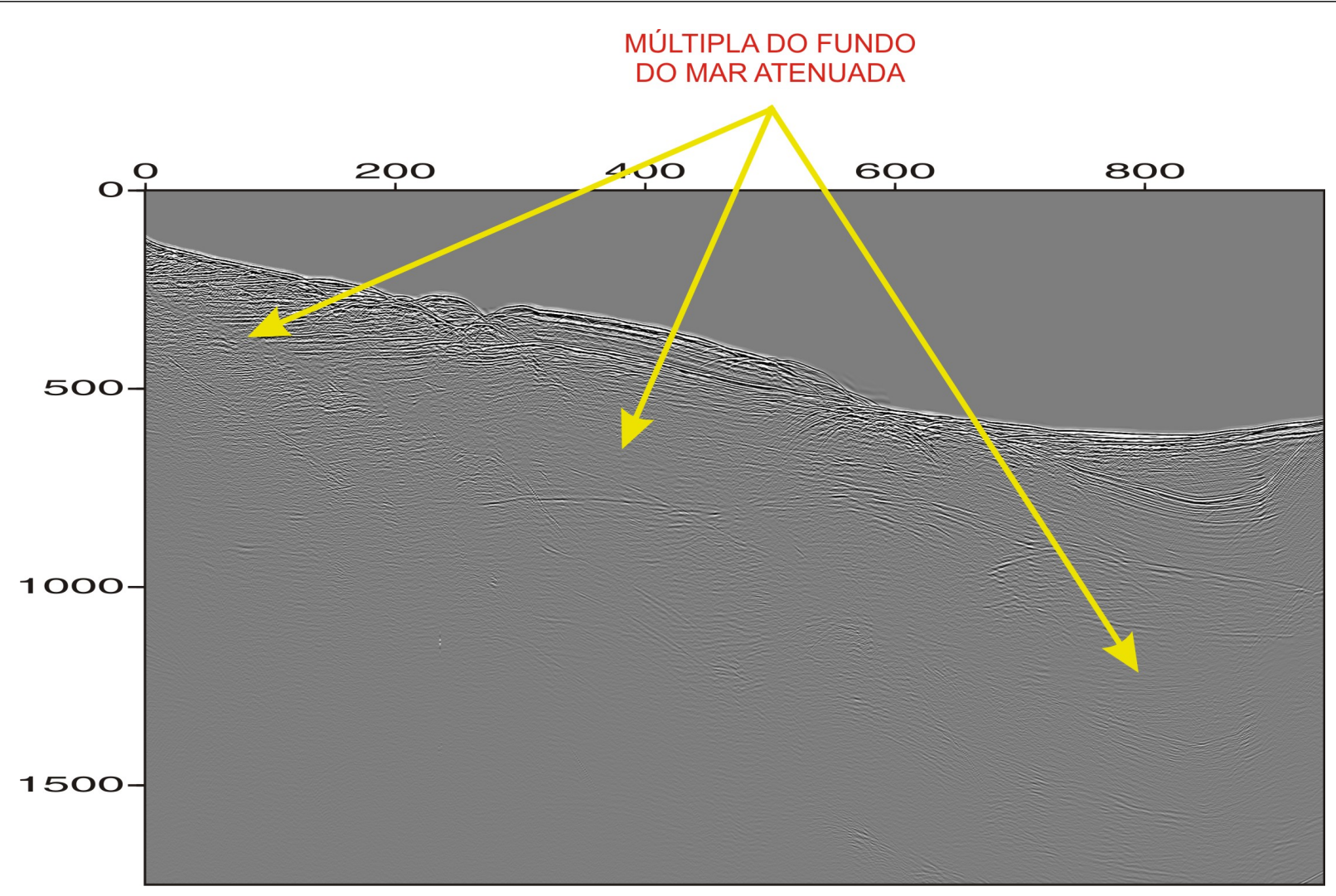

Figura 7: Painel de afastamento comum depois da aplicação da DPM- $L_{2}$.

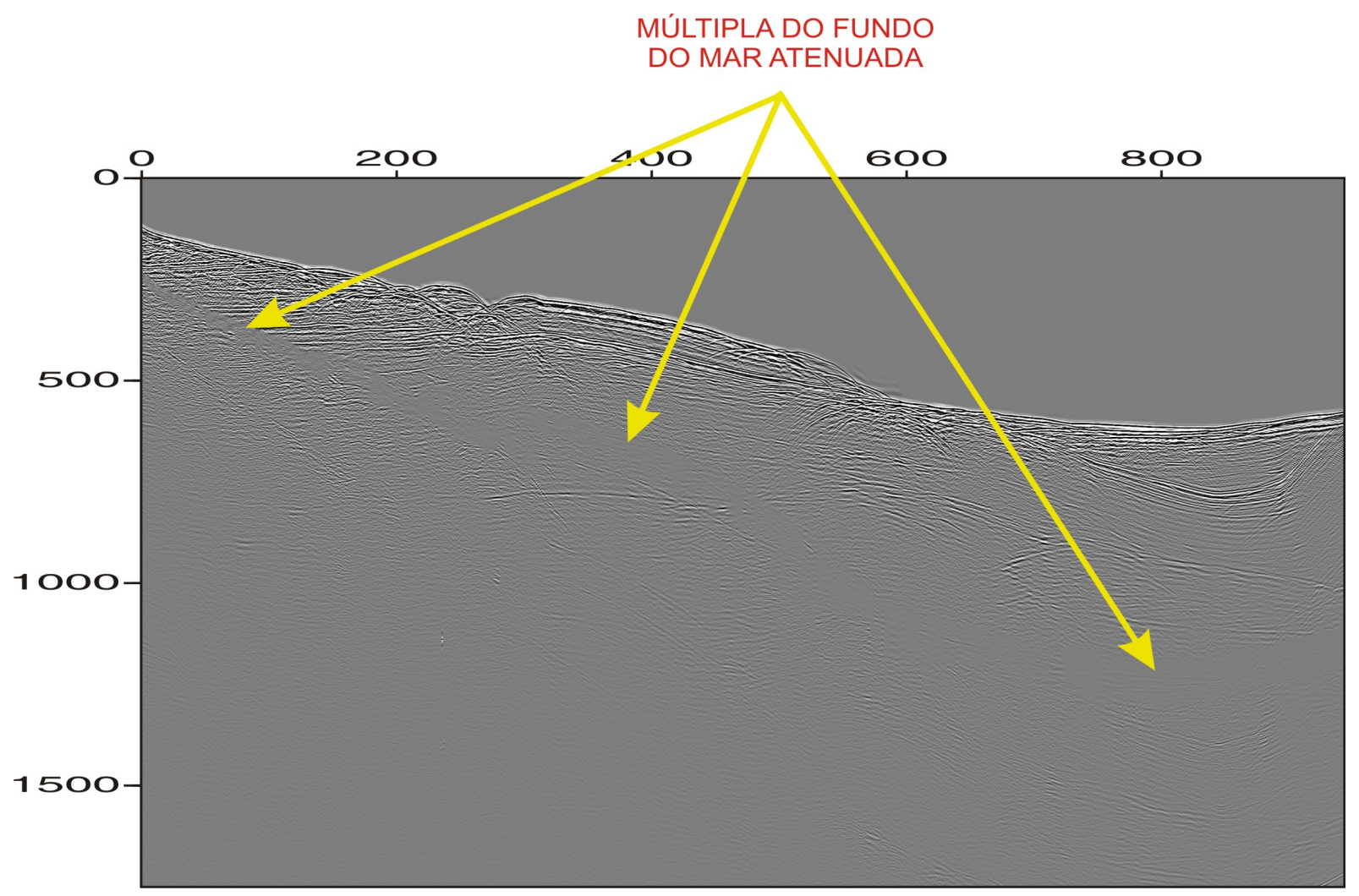

Figura 8: Painel de afastamento comum depois da aplicação da DPM- $L_{p}$. 